\title{
The Burden of Suicide in Rural Bangladesh: Magnitude and Risk Factors
}

\author{
Shumona Sharmin Salam ${ }^{1, *}$, Olakunle Alonge ${ }^{2}$, Md Irteja Islam $^{1}$, Dewan Md Emdadul Hoque ${ }^{1}$ (D), \\ Shirin Wadhwaniya ${ }^{2}$, Md Kamran Ul Baset ${ }^{3}$, Saidur Rahman Mashreky ${ }^{3}$ (i) and Shams El Arifeen ${ }^{1}$ \\ 1 International Centre for Diarrhoeal Disease Research, GPO Box 128, Dhaka 1000, Bangladesh; \\ irteja.islam@icddrb.org (M.I.I.); emdad@icddrb.org (D.M.E.H.); shams@icddrb.org (S.E.A.) \\ 2 Department of International Health, Johns Hopkins University Bloomberg School of Public Health, \\ Baltimore, MA 21205, USA; oalonge1@jhu.edu (O.A.); swadhwa2@jhu.edu (S.W.) \\ 3 Center for Injury Prevention and Research, House \# B-162, Road \# 23, New DOHS, Mohakhali, Dhaka 1206, \\ Bangladesh; kamran_baset@yahoo.co.uk (M.K.U.B.); mashreky@ciprb.org (S.R.M.) \\ * Correspondence: shumona@icddrb.org
}

Received: 5 July 2017; Accepted: 6 September 2017; Published: 9 September 2017

\begin{abstract}
The aim of the paper is to quantify the burden and risk factors of fatal and non-fatal suicidal behaviors in rural Bangladesh. A census was carried out in seven sub-districts encompassing 1.16 million people. Face-to-face interviews were conducted at the household level. Descriptive analyses were done to quantify the burden and Poisson regression was run to determine on risk factors. The estimated rates of fatal and non-fatal suicide were 3.29 and 9.86 per 100,000 person years (PY) observed, respectively. The risk of suicide was significantly higher by 6.31 times among 15-17 and 4.04 times among 18-24 olds compared to 25-64 years old. Married adolescents were 22 times more likely to commit suicide compared to never-married people. Compared to Chandpur/Comilla district, the risk of suicide was significantly higher in Narshingdi. Students had significantly lower risk of non-fatal suicidal behavior compared to skilled laborers. The risk of non-fatal suicidal behavior was lower in Sherpur compared to Chandpur/Comilla. Among adolescents, unskilled laborers were 16 times more likely to attempt suicide than students. The common methods for fatal and non-fatal suicidal behaviors were hanging and poisoning. Suicide is a major public health problem in Bangladesh that needs to be addressed with targeted interventions.
\end{abstract}

Keywords: suicide; attempted suicide; burden; risk factors; rural; Bangladesh; injury; violence

\section{Introduction}

According to the 2014 WHO Global Health Estimates, there were about 803,900 suicides in 2012 representing $1.4 \%$ of the global burden of disease or over 39 million disability adjusted life years (DALYs) lost [1]. Fatal suicidal behavior or suicide is death resulting from self-harm. Worldwide, suicide accounted for $16 \%$ of injury mortality and $1.4 \%$ of total mortality in 2012, making it the 15th most common cause of death for all age groups in that year [1,2]. Analysis of trends indicate that the overall suicide rate has decreased significantly over the past decade. The global suicide rate was 11.4 per 100,000 population in 2012, a decrease from 14.4 per 100,000 in 2000 [1]. However, the rate of decline flat-lined in the latter part of that decade, and the rate in $2008(11.6$ per 100,000) was about the same as rate reported for 2012 [3-5]. Globally, suicide remains the second leading cause of death among 15-29 year olds [1,2]. In addition to these striking facts for suicide, there are indications that, for every adult that dies by suicide, there are 10-20 more who attempted a suicide event [2,5]. This assumption for suicide attempts is based on the WHO world mental health surveys among adults ( $>18$ years of age) in 21 low-middle and high-income countries who report a prevalence of 3-4 per 1000 individuals [6]. 
Fatal and non-fatal suicidal behaviors have been found be mostly prevalent among the vulnerable populations of the world where availability of and accessibility to resources and services for identification and treatment are scarce [2]. In fact, three-fourths of the global suicides have been estimated to occur in low and middle-income countries (LMICs) [2]. The southeast Asia region alone accounts for $40 \%$ of the global suicide deaths, with China and India being the leading contributors [2,4]. Although there are variations in trends and risk factors of suicide among countries in the southeast Asia region, compared to Western countries, the region as whole has higher suicide rates, lower gender (male-to-female) suicide rate gap, higher rates among the elderly, and an increasing trend of youth suicides [5,7]. Reviews and epidemiological data also indicate that there exists wide urban-rural disparity in suicide, with suicide rates often greater in rural areas $[5,8,9]$.

Like most southeast Asian countries, a fundamental challenge for Bangladesh is the lack of quality suicide data or system for monitoring and surveillance. In Bangladesh, a limited number of studies have attempted to quantify suicide rates. Mashreky et al. suggested that about 10,000 people commit suicide in the country in a year [10], and the rate of suicide was found to be 7.3 per 100,000 population (6.5 in males and 8.2 in females) and was highest in the 60+ age group and considerably high among adolescents [10]. These estimates for were based on the 2003 Bangladesh Health and Injury Survey [10]. According to WHO Global Health Estimates, the suicide rate for 2012 in Bangladesh was 7.8 per 100,000 population (8.7 in females and 6.8 in males) [11]. Demographic and health surveillance in two rural sub-districts of Bangladesh between 2004 and 2010 revealed that the most common cause of death for young adults (aged 15-49) was injury (23.5\%) with suicide accounting for $11.9 \%$ [12]. Other studies that have attempted to determine the causes of female deaths, revealed a growing rate of suicide among adolescent females [13]. Analysis of results from national household surveys in 2001 and 2010 and hospital based surveys in 1996-1997 suggested that suicide was the main cause for deaths among adolescent females, accounting for $16-22 \%$ of all deaths in this age group [13]. There is a need for recent population-based data that could be used to define the burden and epidemiology of suicide in Bangladesh, and to consequently address interventions for reducing rates of fatal and non-fatal suicidal behavior in Bangladesh.

The objective of this paper is to quantify the burden and socio-demographic risk factors of fatal and non-fatal suicidal behaviors in rural Bangladesh. The goal is to identify high-risk subgroups, demonstrate any transition in causes of death in the country and to call for action on the need to recognize and address this enormous public health issue on a national scale. It is hoped that this paper will provide evidence to influence national policies to address and invest in related research and preventive approaches.

\section{Materials and Methods}

\subsection{Study Design, Area and Population}

The data for this paper is from an implementation research study conducted with an aim to reduce drowning in under-five children in five districts of rural Bangladesh [14,15]. A cross-sectional baseline census was conducted over a six months period from June through November 2013 before the start of the implementation of the drowning prevention interventions [16]. The census covered 1.165 million people in 51 unions from seven sub-districts of Bangladesh (and this study describing the epidemiology of fatal and nonfatal suicide behaviors is based on the census). The sub-districts included were Matlab North, Matlab South, Daudkandi, Chandpur Sadar, and Manohardi in the central section of the country, and Raiganj and Sherpur in the north.

\subsection{Questionnaire and Data Collection}

The baseline census collected information on fatal and non-fatal injury outcomes including fatal and non-fatal suicidal behaviors, characteristics of the underlying injury mechanisms and health seeking behavior for the injury or death outcome on all populations in the census area. In addition, 
the census collected information on social and demographic characteristics, physical environment and birth history. This was done using a questionnaire covering seven modules (I. Household characteristics and socioeconomic census; II. Birth history; III. Household environment, IV. Death confirmation; V. Injury morbidity; VI. Injury mortality and VII. Injury mechanisms). The injury mechanisms module (module VII) comprised of 12 forms covering 12 injury events, the first of which was attempted suicide. In addition, there were three notification forms: injury notification, death notification and child notification, which provided notifications of any injuries or deaths including suicide and attempted suicide from module I. All fatal injury information (including fatal suicidal behavior) was collected over a one-year recall period, whereas non-fatal injuries (including non-fatal suicidal behavior) were collected over a six-month recall period.

Suicide (or non-fatal suicidal behavior) was operationally defined as a self-initiated injury event that resulted in a fatal outcome (or non-fatal outcome). The injury events assessed were defined based on ICD-10 classification, and are described in details elsewhere [16].

All information was collected directly from the head of households or any adults 18 years above with sufficient knowledge of the household. All forms were written in English and translated to Bangla. The forms were back-translated and pilot-tested prior to the actual data collection.

Data collection was implemented by two sets of trained data collectors. The first set of data collectors completed the questionnaire forms in module I-III and notified of any fatal suicide or non-fatal suicide events. In module I after enlisting all the household members and their soico-demographic information, we asked "whether the member had suffered from any non-fatal injuries (read out all injuries) in the past six months". In order to obtain information on deaths we asked "whether there were any deaths in the past one year". Based on the notification, a second set of data collectors completed the questionnaire forms in module IV-VII only in households with the suicide injury or death event. Each set of the questionnaire forms took between 40-50 min to complete. Written informed consent was obtained prior to data collection.

\subsection{Statistical Method and Analyses}

Descriptive statistics were used to describe the population by various variables such as age, gender, marital status, educational attainment, wealth quintile and geographical area. The overall rates of suicide and attempted suicide was calculated per 100,000 person years observed (PYO). The mortality and morbidity rates were also stratified by the above-mentioned variables. Principal component analysis was done to estimate the wealth quintile using various variables that indicate asset availability and housing conditions.

Multivariable analysis was performed with multilevel Poisson regression models. Model estimates were reported as incidence rate ratios (IRR), with their respective $95 \%$ confidence intervals. The models were first implemented with one covariate, and then adjusted for other covariates and possible confounders (age, gender, marital status, educational attainment, wealth quintile and geographical area). Age, education and occupation were considered as categorical variables, whereas gender was considered as a binary predictor (male as reference group). Socioeconomic quintile was considered as ordinal data from lowest to highest. Age was theorized to modify the risk of fatal and non-fatal suicidal behavior with regards to other variables, especially marital status in rural Bangladesh. Thus, separate analyses were implemented for (1) individuals 15 years and older, and (2) adolescents-only (aged 10-17 years) to adjust for the possible interactions with age. The final results for individuals above 15 years of age are presented in the paper, and results for the adolescent-only analyses have been included in the (Supplementary Materials Tables S1 and S2).

Variable construction and estimations were done with the statistical software STATA 13 (Stata Corp. 2013. Stata Statistical Software: Release 13. College Station, TX, USA: Stata Corp LP.). 


\subsection{Ethical Statement}

Ethical approval was obtained from of the Institutional Review Board of the Johns Hopkins Bloomberg School of Public Health (JHSPH) (ethics approval code is 00004746); and Ethics Review Committees of International Centre for Diarrhoeal Disease Research, Bangladesh (icddr,b) and Center for Injury Prevention and Research Bangladesh (CIPRB) under the study Saving of Lives from Drowning (SoLiD), Bangladesh.

\section{Results}

The survey covered a population of 1,169,593 (Table 1). Overall, $21.6 \%$ of the population was less than 10 years of age, $72.3 \%$ were between $10-65$ years of age and only $6.1 \%$ of the population was above 65 years. More than three-fifth $(65 \%)$ of the respondents had received at least primary education and about half $(48.8 \%)$ of the population were married during the time of the survey. Three-fourths $(74.6 \%)$ of the population were not employed and were either retired, unemployed, housewives, students or children. Among those who were employed, a majority were involved in agricultural activities $(9 \%)$, followed by skilled work (7.6\%) and business (5.3\%). Household wealth quintiles were evenly distributed. The majority of respondents resided in Matlab North (22.7), Sherpur (19.5\%), Matlab South $(17.9 \%)$ and Manohardi (17.5\%) sub-districts of the country.

Table 1. Socio-demographic characteristics of the respondents.

\begin{tabular}{|c|c|c|}
\hline Characteristics & $N=1,169,593$ & $\%$ \\
\hline \multicolumn{3}{|l|}{ Age (in years) } \\
\hline$<10$ & 252,392 & 21.6 \\
\hline $10-14$ & 142,121 & 12.2 \\
\hline $15-17$ & 62,098 & 5.3 \\
\hline $18-24$ & 133,534 & 11.4 \\
\hline $25-64$ & 508,059 & 43.4 \\
\hline $65+$ & 71,389 & 6.1 \\
\hline \multicolumn{3}{|l|}{ Sex } \\
\hline Male & 567,674 & 48.5 \\
\hline Female & 601,919 & 51.5 \\
\hline \multicolumn{3}{|l|}{ Education $^{1}$} \\
\hline No education & 295,314 & 25.3 \\
\hline Primary complete (five years) & 407,923 & 34.9 \\
\hline Secondary complete (10 years) & 289,658 & 24.8 \\
\hline Secondary and above & 63,873 & 5.5 \\
\hline Under five children & 112,664 & 9.6 \\
\hline \multicolumn{3}{|l|}{ Occupation $^{2}$} \\
\hline Agriculture & 104,956 & 9.0 \\
\hline Business & 61,661 & 5.3 \\
\hline Skilled labor & 89,151 & 7.6 \\
\hline Unskilled labor & 24,520 & 2.1 \\
\hline Transport worker & 17,037 & 1.5 \\
\hline Students & 312,537 & 26.7 \\
\hline Retired/unemployed/housewife & 408,583 & 35.0 \\
\hline Children & 144,454 & 12.4 \\
\hline Others (NA) & 5948 & 0.5 \\
\hline \multicolumn{3}{|l|}{ Marital Status } \\
\hline Married (Reference) & 571,206 & 48.8 \\
\hline Never-married & 227,319 & 19.4 \\
\hline Widowed/Divorced/Separated & 59,033 & 5.0 \\
\hline Children $(<12$ years $)$ & 312,035 & 26.7 \\
\hline
\end{tabular}


Table 1. Cont.

\begin{tabular}{lcc}
\hline \multicolumn{1}{c}{ Characteristics } & $N=\mathbf{1 , 1 6 9 , 5 9 3}$ & $\%$ \\
\hline Wealth quintile & & \\
\hline Lowest & 211,601 & 18.1 \\
Second & 218,695 & 18.7 \\
Middle & 238,371 & 20.4 \\
Fourth & 247,716 & 21.2 \\
Highest & 253,210 & 21.6 \\
\hline Sub-district & & \\
\hline Matlab North & 265,897 & 22.7 \\
Matlab South & 209,772 & 17.9 \\
Chandpur Sadar & 128,356 & 11.0 \\
Raiganj & 104,357 & 8.9 \\
Sherpur & 228,519 & 19.5 \\
Manohardi & 204,319 & 17.5 \\
Daudkandi & 28,373 & 2.4 \\
\hline District & & \\
\hline Chandpur/Comilla & 632,398 & 54.1 \\
Sirajganj & 104,357 & 8.9 \\
Sherpur & 228,519 & 19.5 \\
Narshingdi & 204,319 & 17.5 \\
\hline
\end{tabular}

${ }^{1}$ Information on education missing for 0.01 (161) participants; ${ }^{2}$ Information on occupation missing for 0.06 (746) participants.

A total of 38 fatal suicide and 57 non-fatal suicidal events were recorded in the past one year and six months prior to the survey, respectively (Table 2). In rural Bangladesh, the estimated rates of fatal and non-fatal suicidal behavior were 3.29 per 100,000 PYO and 9.86 per 100,000 PYO. Suicide rates were found to be higher among the younger population specifically the adolescents compared to the adults and the elderly. The rates were highest among 15-17 year olds (11.3 per 100,000 PYO), followed by 18-24 year olds (7.5 per 100,000 PYO) and 3.52 per 100,000 PYO among young adolescents (aged between 10-14 years old). There was no difference in the rates of fatal suicidal behavior comparing males and females of all ages, and the gender ratio (male-to-female) was 1.18. The rates did not vary much by marital status and no definitive pattern in suicidal behavior was observed by education or wealth quintile. The rate was highest among those who had completed 10 years of education or had no education, and among those belonging to the middle wealth quintile. The suicide rate was found to be high among the transport workers (17.66 per 100,000 PYO), but was similar among other occupations, ranging from 3.38 in skilled labors to 4.80 in those involved in agricultural activities. Geographically, suicide rates were found to be higher in Raiganj and Manohardi Sub-districts in Northern Bangladesh compared to the sub-districts of Matlab situated in central Bangladesh. 
Table 2. Distribution of suicide and attempted suicide rates by socio-demographic and geographical factors, rural Bangladesh.

\begin{tabular}{|c|c|c|c|c|c|c|c|}
\hline \multirow[b]{2}{*}{ Characteristics } & \multirow[b]{2}{*}{$N$} & \multicolumn{3}{|c|}{ Fatal Suicidal Behavior } & \multicolumn{3}{|c|}{ Non-Fatal Suicidal Behavior } \\
\hline & & $\begin{array}{c}\text { Person Years } \\
\text { Observed (PYO) }\end{array}$ & Frequency & $\begin{array}{c}\text { Mortality Rate } \\
\text { per 100,000 } \\
\text { Person Years } \\
\text { Observed (PYO) }\end{array}$ & $\begin{array}{c}\text { Person Years } \\
\text { Observed (PYO) }\end{array}$ & Frequency & $\begin{array}{c}\text { Morbidity Rate } \\
\text { per 100,000 } \\
\text { Person Years } \\
\text { Observed (PYO) }\end{array}$ \\
\hline Total & $1,169,593$ & $1,153,901$ & 38 & 3.29 & 578,046 & 57 & 9.86 \\
\hline \multicolumn{8}{|l|}{ Age (in years) } \\
\hline$<10$ & 252,392 & 240,739 & 0 & 0.00 & 122,966 & 0 & 0.00 \\
\hline 10-14 & 142,121 & 141,849 & 5 & 3.52 & 70,894 & 4 & 5.64 \\
\hline $15-17$ & 62,098 & 61,972 & 7 & 11.30 & 30,965 & 4 & 12.92 \\
\hline $18-24$ & 133,534 & 133,247 & 10 & 7.50 & 66,568 & 13 & 19.53 \\
\hline $25-64$ & 508,059 & 506,348 & 14 & 2.76 & 252,581 & 34 & 13.46 \\
\hline $65+$ & 71,389 & 69,745 & 2 & 2.87 & 34,072 & 2 & 5.87 \\
\hline \multicolumn{8}{|l|}{ Sex } \\
\hline Male & 567,674 & 559,566 & 20 & 3.57 & 280,538 & 28 & 9.98 \\
\hline Female & 601,919 & 594,335 & 18 & 3.03 & 297,508 & 29 & 9.75 \\
\hline \multicolumn{8}{|l|}{ Education $^{1}$} \\
\hline No education & 295,314 & 293,176 & 11 & 3.75 & 145,672 & 16 & 10.98 \\
\hline Primary complete (five years) & 407,923 & 406,727 & 9 & 2.21 & 203,011 & 17 & 8.37 \\
\hline Secondary complete (10 years) & 289,658 & 288,858 & 17 & 5.89 & 144,209 & 22 & 15.26 \\
\hline Secondary and above & 63,873 & 63,690 & 1 & 1.57 & 31,787 & 2 & 6.29 \\
\hline Under five children & 112,664 & 101,294 & 0 & 0.00 & 53,291 & 0 & 0.00 \\
\hline \multicolumn{8}{|l|}{ Occupation $^{2}$} \\
\hline Agriculture & 104,956 & 104,221 & 5 & 4.80 & 51,802 & 11 & 21.23 \\
\hline Business & 61,661 & 61,397 & 0 & 0.00 & 30,600 & 1 & 3.27 \\
\hline Skilled labor & 89,151 & 88,864 & 3 & 3.38 & 44,330 & 8 & 18.05 \\
\hline Unskilled labor & 24,520 & 24,417 & 1 & 4.10 & 12,169 & 4 & 32.87 \\
\hline Transport worker & 17,037 & 16,989 & 3 & 17.66 & 8480 & 1 & 11.79 \\
\hline Students & 312,537 & 311,935 & 12 & 3.85 & 155,892 & 6 & 3.85 \\
\hline Retired/Unemployed/Housewife & 408,583 & 406,478 & 14 & 3.44 & 202,394 & 26 & 12.85 \\
\hline Children (Under 12 years) & 144,454 & 133,003 & 0 & 0.00 & 69,125 & 0 & 0.00 \\
\hline Others (NA) & 5948 & 5857 & 0 & 0.00 & 2886 & 0 & 0.00 \\
\hline
\end{tabular}


Table 2. Cont

\begin{tabular}{|c|c|c|c|c|c|c|c|}
\hline \multirow[b]{2}{*}{ Characteristics } & \multirow[b]{2}{*}{$N$} & \multicolumn{3}{|c|}{ Fatal Suicidal Behavior } & \multicolumn{3}{|c|}{ Non-Fatal Suicidal Behavior } \\
\hline & & $\begin{array}{c}\text { Person Years } \\
\text { Observed (PYO) }\end{array}$ & Frequency & $\begin{array}{c}\text { Mortality Rate } \\
\text { per 100,000 } \\
\text { Person Years } \\
\text { Observed (PYO) }\end{array}$ & $\begin{array}{c}\text { Person Years } \\
\text { Observed (PYO) }\end{array}$ & Frequency & $\begin{array}{c}\text { Morbidity Rate } \\
\text { per 100,000 } \\
\text { Person Years } \\
\text { Observed (PYO) }\end{array}$ \\
\hline \multicolumn{8}{|l|}{ Marital Status } \\
\hline Married & 571,206 & 568,601 & 25 & 4.40 & 283,292 & 37 & 13.06 \\
\hline Never-married & 227,319 & 226,802 & 11 & 4.85 & 113,320 & 15 & 13.24 \\
\hline Widowed/Divorced/Separated & 59,033 & 58,192 & 0 & 0.00 & 28,705 & 3 & 10.45 \\
\hline Children (Under 12 years) & 312,035 & 300,306 & 2 & 0.67 & 152,729 & 2 & 1.31 \\
\hline \multicolumn{8}{|l|}{ Wealth quintile } \\
\hline Lowest & 211,601 & 208,601 & 7 & 3.36 & 104,479 & 10 & 9.57 \\
\hline Second & 218,695 & 215,949 & 5 & 2.32 & 108,190 & 13 & 12.02 \\
\hline Middle & 238,371 & 235,239 & 10 & 4.25 & 117,830 & 14 & 11.88 \\
\hline Fourth & 247,716 & 244,482 & 9 & 3.68 & 122,460 & 11 & 8.98 \\
\hline Highest & 253,210 & 249,630 & 7 & 2.80 & 125,088 & 9 & 7.19 \\
\hline \multicolumn{8}{|l|}{ Sub-district } \\
\hline Matlab North & 265,897 & 262,510 & 7 & 2.67 & 131,406 & 11 & 8.37 \\
\hline Matlab South & 209,772 & 206,600 & 5 & 2.42 & 103,492 & 14 & 13.53 \\
\hline Chandpur Sadar & 128,356 & 126,659 & 3 & 2.37 & 63,475 & 5 & 7.88 \\
\hline Raiganj & 104,357 & 103,052 & 5 & 4.85 & 51,612 & 11 & 21.31 \\
\hline Sherpur & 228,519 & 225,476 & 6 & 2.66 & 113,055 & 4 & 3.54 \\
\hline Manohardi & 204,319 & 201,641 & 12 & 5.95 & 101,004 & 12 & 11.88 \\
\hline Daudkandi & 28,373 & 27,962 & 0 & 0.00 & 14,002 & 0 & 0.00 \\
\hline \multicolumn{8}{|l|}{ District } \\
\hline Chandpur/Comilla & 632,398 & 623,732 & 15 & 2.40 & 312,375 & 30 & 9.60 \\
\hline Sirajganj & 104,357 & 103,052 & 5 & 4.85 & 51,612 & 11 & 21.31 \\
\hline Sherpur & 228,519 & 225,476 & 6 & 2.66 & 113,055 & 4 & 3.54 \\
\hline Narshingdi & 204,319 & 201,641 & 12 & 5.95 & 101,004 & 12 & 11.88 \\
\hline
\end{tabular}

${ }^{1}$ Information on education missing for 0.01 (161) participants; ${ }^{2}$ Information on occupation missing for 0.06 (746) participants. 
Table 3 presents stratified analysis of suicide rates by age and sex. Among males, suicide rates are highest among the 15-17 year olds followed by 10-14 year olds, whereas among females the rates are highest among the 15-17 year olds and 18-24 year olds. The rates of suicide are higher in females among 15-24 year olds than in males of the same age group. The rates are, however, higher in males for 10-14 and 25-64 year olds.

Table 3. Suicide rates by age and sex, rural Bangladesh.

\begin{tabular}{ccccccccc}
\hline $\begin{array}{c}\text { Age } \\
\begin{array}{c}\text { Group } \\
\text { (Years) }\end{array}\end{array}$ & PYO & $\begin{array}{c}\text { Suicide } \\
(\boldsymbol{n})\end{array}$ & $\begin{array}{c}\text { Mortality } \\
\text { Rate/100,000 } \\
\text { PYO }\end{array}$ & $\begin{array}{c}\text { Confidence } \\
\text { Interval (CI) }\end{array}$ & PYO & $\begin{array}{c}\text { Suicide } \\
(\boldsymbol{n})\end{array}$ & $\begin{array}{c}\text { Mortality } \\
\text { Rate/100,000 } \\
\text { PYO }\end{array}$ & $\begin{array}{c}\text { Confidence } \\
\text { Interval (CI) }\end{array}$ \\
\hline$<10$ & 122,970 & 0 & 0.00 & & 117,769 & 0 & 0.00 & \\
$10-14$ & 72,339 & 4 & 5.53 & $1.51-14.16$ & 69,510 & 1 & 1.44 & $0.04-8.02$ \\
$15-17$ & 33,156 & 3 & 9.05 & $1.87-26.44$ & 28,815 & 4 & 13.88 & $3.78-35.54$ \\
$18-24$ & 57,909 & 3 & 5.18 & $1.07-15.14$ & 75,339 & 7 & 9.29 & $3.74-19.14$ \\
$25-64$ & 236,636 & 9 & 3.80 & $1.74-7.22$ & 269,712 & 5 & 1.85 & $0.60-4.33$ \\
$65+$ & 36,556 & 1 & 2.74 & $0.07-15.24$ & 33,189 & 1 & 3.01 & $0.08-16.79$ \\
\hline Total & 559,566 & 20 & 3.57 & $2.18-5.52$ & 594,335 & 18 & 3.03 & $1.79-4.79$ \\
\hline
\end{tabular}

In contrast to suicide rates, the rates of non-fatal suicidal behavior were highest among individuals aged 18-24 year olds (19.53 per 100,000 PYO), 25-64 year olds (13.46 per 100,000 PYO) followed by 15-17 year olds (12.92 per 100,000 PYO). The rates were highest in people involved in un-skilled labor (32.87 per 100,000 PYO) and agricultural activities (21.23 per 100,000 PYO) and more common in people belonging to the bottom three wealth quintiles. Non-fatal suicidal behavior was also evident among women who were widowed, divorced or separated (10.45 per 100,000 PYO), which was not present in the case of suicide. Along with Raiganj (21.31 per 100,000 PYO) and Manohardi (11.88 per 100,000 PYO) sub-districts, the rates were also high in Matlab South (13.53 per 100,000 PYO).

Table 4 presents crude and adjusted IRR for fatal suicide and non-fatal suicidal behavior among respondents aged 15 years or more in the study. The significant factors associated with fatal suicidal behavior in the adjusted model included age, and geographical region (Table 4). The adjusted risk of fatal suicidal behavior was clearly higher, by six and four times among the 15-17 year olds (IRR 6.31, CI 1.35-29.32) and 18-24 year olds (IRR 4.04, CI 1.56-10.47) respectively, compared to those aged between 25-64 years old. In addition, the risk was significantly higher in the Narshingdi district (IRR 2.89, CI 1.26-6.65) compared to Chandpur/Comilla district. Although transport workers were more likely to commit suicide compared to skilled labor, the relationship was not significant in the adjusted model. In addition, adjusted analysis only among adolescents aged between 10-17 year olds revealed that the risk of fatal suicidal behavior was 22 times higher among those who were married compared to those who were never-married (IRR 22.06, CI 3.70-131.63) (Supplementary Materials Table S1).

Table 4. Unadjusted and adjusted analysis for fatal suicidal behavior by socio-demographic and geographical factors, rural Bangladesh.

\begin{tabular}{lcccc}
\hline \multirow{2}{*}{ Characteristics } & \multicolumn{2}{c}{ Unadjusted } & \multicolumn{2}{c}{ Adjusted } \\
\cline { 2 - 5 } & $\begin{array}{c}\text { Incidence Rate } \\
\text { Ratio (IRR) }\end{array}$ & $\mathbf{9 5 \%}$ CI & $\begin{array}{c}\text { Incidence Rate } \\
\text { Ratio (IRR) }\end{array}$ & 95\% CI \\
\hline Age (in years) & & & & \\
\hline $15-17$ & $4.09 * *$ & $1.64-10.12$ & $6.31 *$ & $1.35-29.32$ \\
$18-24$ & $2.71^{*}$ & $1.2-6.11$ & $4.04 *$ & $1.56-10.47$ \\
$25-64$ & Reference & & Reference & \\
$65+$ & 1.04 & $0.24-4.56$ & 1.48 & $0.32-6.87$ \\
\hline
\end{tabular}


Table 4. Cont.

\begin{tabular}{|c|c|c|c|c|}
\hline \multirow[b]{2}{*}{ Characteristics } & \multicolumn{2}{|c|}{ Unadjusted } & \multicolumn{2}{|c|}{ Adjusted } \\
\hline & $\begin{array}{l}\text { Incidence Rate } \\
\text { Ratio (IRR) }\end{array}$ & $95 \%$ CI & $\begin{array}{c}\text { Incidence Rate } \\
\text { Ratio (IRR) }\end{array}$ & $95 \%$ CI \\
\hline \multicolumn{5}{|l|}{ Sex } \\
\hline Male & 1.05 & $0.53-2.08$ & 0.87 & $0.29-2.56$ \\
\hline Female & Reference & & Reference & \\
\hline \multicolumn{5}{|l|}{ Education } \\
\hline No education & 2.81 & $0.36-21.75$ & 5.48 & $0.56-53.10$ \\
\hline Primary complete (five years) & 1.50 & $0.17-12.83$ & 2.27 & $0.22-22.62$ \\
\hline Secondary complete (10 years) & 4.15 & $0.54-31.25$ & 4.04 & $0.49-32.97$ \\
\hline Secondary and above & Reference & & Reference & \\
\hline \multicolumn{5}{|l|}{ Occupation } \\
\hline Agriculture & 1.40 & $0.33-5.84$ & 0.99 & $0.22-4.45$ \\
\hline Business & 0.00 & & 0.00 & \\
\hline Skilled labor & Reference & & Reference & \\
\hline Unskilled labor & 1.25 & $0.13-12.01$ & 1.20 & $0.12-11.82$ \\
\hline Transport worker & $5.14 *$ & $1.03-25.48$ & 4.25 & $0.82-21.82$ \\
\hline Students & 2.89 & $0.76-10.88$ & 1.86 & $0.32-10.67$ \\
\hline Retired/Unemployed/Housewife & 0.95 & $0.27-3.33$ & 0.62 & $0.13-2.87$ \\
\hline Children (Under 12 years) & 0.00 & & 0.00 & \\
\hline Others (NA) & 0.00 & & 0.00 & \\
\hline \multicolumn{5}{|l|}{ Marital Status } \\
\hline Married & 0.68 & $0.31-1.47$ & 3.60 & $0.92-14.06$ \\
\hline Never-married & Reference & & Reference & \\
\hline Widowed/Divorced/Separated & 0.00 & & 0.00 & \\
\hline \multicolumn{5}{|l|}{ Wealth quintile } \\
\hline Lowest & Reference & & Reference & \\
\hline Second & 0.76 & $0.20-2.83$ & 0.76 & $0.20-2.85$ \\
\hline Middle & 1.55 & $0.52-4.61$ & 1.71 & $0.55-5.26$ \\
\hline Fourth & 1.44 & $0.48-4.28$ & 1.61 & $0.51-5.07$ \\
\hline Highest & 0.92 & $0.28-3.01$ & 1.03 & $0.28-3.71$ \\
\hline \multicolumn{5}{|l|}{ District } \\
\hline Chandpur/Comilla & Reference & & Reference & \\
\hline Sirajganj & 2.48 & $0.87-7.02$ & 2.32 & $0.77-6.88$ \\
\hline Sherpur & 1.19 & $0.41-3.37$ & 0.97 & $0.33-2.89$ \\
\hline Narshingdi & 2.83 * & $1.24-6.42$ & $2.89 *$ & $1.26-6.65$ \\
\hline
\end{tabular}

The two factors that were significantly associated with non-fatal suicidal behavior in both the adjusted model among respondents aged 15 years or older included occupation and districts (Table 5). Compared to those involved in skilled labor, students were found to have a significantly lower risk (adjusted) of non-fatal suicidal behavior (IRR 0.21, CI 0.04-1.00). People living in Sherpur were found to be at significantly lower risk compared to those living in Chandpur/Comilla sub-districts (IRR 0.28, CI 0.08-0.94). Although, the risk of non-fatal suicidal behavior was twice among people living in Sirajganj district compared to those living in Chandpur/Comilla districts (IRR 2.10, CI 1.01-4.36) in the unadjusted model, the relationship was not significant in the adjusted model. Among adolescents aged 10-17 years, the risk of non-fatal suicidal behavior (adjusted) was 16 times higher among those involved in unskilled labor compared to students (IRR 15.75. CI 1.35-184.52) (Supplementary Materials Table S2). 
Table 5. Unadjusted and adjusted analysis of non-fatal suicidal behavior by socio-demographic and geographical factors, rural Bangladesh.

\begin{tabular}{|c|c|c|c|c|}
\hline \multirow[b]{2}{*}{ Characteristics } & \multicolumn{2}{|c|}{ Unadjusted } & \multicolumn{2}{|c|}{ Adjusted } \\
\hline & $\begin{array}{c}\text { Incidence Rate } \\
\text { Ratio (IRR) }\end{array}$ & $95 \%$ CI & $\begin{array}{c}\text { Incidence Rate } \\
\text { Ratio (IRR) }\end{array}$ & $95 \%$ CI \\
\hline \multicolumn{5}{|l|}{ Age (in years) } \\
\hline $15-17$ & 0.97 & $0.34-2.72$ & 1.01 & $0.25-4.03$ \\
\hline $18-24$ & 1.46 & $0.77-2.77$ & 1.31 & $0.59-2.91$ \\
\hline $25-64$ & Reference & & Reference & \\
\hline $65+$ & 0.44 & $0.10-0.82$ & 0.49 & $0.11-2.18$ \\
\hline \multicolumn{5}{|l|}{ Sex } \\
\hline Male & 1.07 & $0.63-1.84$ & 0.63 & $0.24-1.67$ \\
\hline Female & Reference & & Reference & \\
\hline \multicolumn{5}{|l|}{ Education } \\
\hline No education & 1.91 & $0.44-8.35$ & 1.59 & $0.31-8.02$ \\
\hline Primary complete (5 years) & 2.23 & $0.51-9.75$ & 1.60 & $0.45-2.00$ \\
\hline Secondary complete (10 years) & 2.71 & $0.64-11.55$ & 2.28 & $0.66-3.03$ \\
\hline Secondary and above & Reference & & & \\
\hline \multicolumn{5}{|l|}{ Occupation } \\
\hline Agriculture & 1.15 & $0.46-2.87$ & 1.49 & $0.56-3.97$ \\
\hline Business & 0.18 & $0.02-1.43$ & 0.20 & $0.03-1.69$ \\
\hline Skilled labor & Reference & & Reference & \\
\hline Unskilled labor & 1.87 & $0.56-6.21$ & 2.25 & $0.66-7.68$ \\
\hline Transport worker & 0.63 & $0.08-5.10$ & 0.78 & $0.10-6.38$ \\
\hline Students & 0.41 & $0.10-1.54$ & $0.21 *$ & $0.04-1.00$ \\
\hline Retired/Unemployed/Housewife & 0.69 & $0.31-1.53$ & 0.57 & $0.19-1.72$ \\
\hline Children (Under 12 years) & 0.00 & & 0.00 & \\
\hline Others (NA) & 0.00 & & 0.00 & \\
\hline \multicolumn{5}{|l|}{ Marital Status } \\
\hline Married & 0.72 & $0.39-1.37$ & 0.55 & $0.22-1.38$ \\
\hline Never-married & Reference & & Reference & \\
\hline Widowed/Divorced/Separated & 0.58 & $0.17-2.04$ & 0.6 & $0.13-2.86$ \\
\hline Children $<12$ years & 0.00 & & & \\
\hline \multicolumn{5}{|l|}{ Wealth quintile } \\
\hline Lowest & Reference & & Reference & \\
\hline Second & 1.27 & $0.53-3.00$ & 1.17 & $0.49-2.80$ \\
\hline Middle & 1.15 & $0.48-2.71$ & 1.12 & $0.46-2.71$ \\
\hline Fourth & 0.98 & $0.40-2.35$ & 0.99 & $0.40-2.45$ \\
\hline Highest & 0.77 & $0.30-1.94$ & 0.82 & $0.30-2.19$ \\
\hline \multicolumn{5}{|l|}{ District } \\
\hline Chandpur/Comilla & Reference & & Reference & \\
\hline Sirajganj & 2.11 * & $1.02-4.34$ & 2.06 & $0.96-4.42$ \\
\hline Sherpur & 0.31 & $0.09-1.01$ & $0.28 *$ & $0.08-0.94$ \\
\hline Narshingdi & 1.34 & $0.68-2.63$ & 1.34 & $0.67-2.66$ \\
\hline
\end{tabular}

The most common method of suicide was hanging (59\%) followed by poisoning (31\%), burn $(5.1 \%)$, drowning $(2.6 \%)$ and exsanguination $(2.6 \%)$. These frequencies were reversed for non-fatal suicidal behavior, where poisoning $(71.93 \%)$ was the most common method followed by hanging $(22.81 \%)$, implying that the survival rate was higher among those who attempted to use poisoning as the method. Pesticides were the most commonly used poisoning material $(62.7 \%$ in case of non-fatal suicidal behavior and $83 \%$ in case of fatal suicidal behavior). About two-thirds of the cases undertook 
the events at home followed by in-laws and other's house (about one-fifth) and only a small proportion of cases chose public places for the event. There were no differences in the methods and place by gender and the pattern was similar.

\section{Discussion}

This paper set out to explore the epidemiology of fatal and non-fatal suicidal behavior in rural Bangladesh. The estimated rates of fatal and non-fatal suicidal behavior were 3.29 and 9.86 per 100,000 PYO, respectively. The significant factors associated with fatal suicidal behavior included age, marital status and geographical region, whereas the two factors that were significantly associated with non-fatal suicidal behavior were occupation and districts.

The estimated rate for fatal suicidal behavior was 3.29 per 100,000 PYO (3.25 per 100,000 population per year), whereas the rate for non-fatal suicidal behavior was 9.86 per 100,000 PYO (4.87 per 100,000 population per six months) indicating that approximately 5000 people lost their lives to suicide and another 15,000 attempted a non-fatal suicide event in 2013. This rate for fatal suicidal behavior was, however, two times lower than the overall rate presented in the Global Health Estimates for 2012 [1] and almost four times lower than the rate of suicide in rural Bangladesh reported by Mashreky et al. [10]. Perhaps, this may be because the current data is only representative of rural Bangladesh, and significant differences may exist in suicide rates between urban and rural populations in Bangladesh. In addition, the rates are lower than that of other Asian countries such as China (9.8) [8], India (22.0) [9], South Korea (31.0), Japan (24.0), Sri Lanka (23.0), Taiwan (17.6), and Hong Kong (13.8) [5,8]. The rural rates in several of these Asian countries, including China, India, Sri Lanka, Japan, and Taiwan have been found to be higher compared to urban rates $[5,8,9]$. Disaggregation by gender and age reveals further disparity in urban-rural rates in these countries. Several contextual factors have been described to contribute to the increased risk of suicide in rural areas, including socio-economic and cultural differences, availability and accessibility to services, access to means, and, community attitudes towards mental illness and care-seeking [17-19] The rates presented for different countries should be interpreted with caution since only a few countries in the world have good vital registration or data collection systems that help obtain high quality data. For example, estimates in countries such as Hong Kong, Japan, Malaysia, Singapore, South Korea, and Taiwan are considered to be more reliable compared to countries such as China, India, Thailand and Sri Lanka [5]. The situation is further complicated by issues such as stigmatization and illegality that result in misclassification and under-reporting of suicide and suicidal attempts. It must be noted that Bangladesh is predominantly a Muslim country with not only strong religious sanctions against suicide and suicidal attempts but also has punitive laws against attempted suicide (Bangladesh penal code, 1860).

The gender gap (male to female ratio) for suicide was very low at 1.2. The findings contrast with High Income Countries (HIC), where suicide rates for males are about 3-4 times higher than those for females but similar to other Low and Middle Income Countries (LMICs) where the gender gap is low (1.6) $[2,4,20,21]$. Several Asian countries such as China, Hong Kong, Japan, India, Taiwan, Singapore, and Sri Lanka also have a lower gender ratio although there is some evidence that the gap may be increasing $[5,7,8,22,23]$. There are many potential reasons for different suicide rates in men and women: gender equality issues, differences in socially acceptable methods of dealing with stress and conflict for men and women, availability of and preference for different means of suicide, availability and patterns of alcohol consumption, and differences in care-seeking rates for mental disorders between men and women.

Our study also revealed that risks of fatal and non-fatal suicidal behavior were higher among the young, including the adolescents especially those 15-17 year olds, and young adults 18-24 year olds. The rates of suicide varied by age and sex with female 15-24 year olds individuals to be more likely to commit suicide compared to their male counterparts. Several studies in Bangladesh have also attempted to highlight this issue indicating that the young people, and not the elderly, are particularly vulnerable to injuries including self-harm and suicide in the country $[12,13,24-27]$. Despite this, there 
has been no study in Bangladesh that has aimed to determine the factors influencing high suicide rates among adolescents and young adults.

We conducted separate regression analysis among adolescents age 10-17 years and found early marriage to significant stressor for suicide, which explains why the suicide burden affect the female adolescents more. Such high suicide rates among adolescent women may be because of early and forced marriage, marital abuse related to dowry, and complete lack of opportunity for advancement and development coupled with the tendency to impulsive behavior and actions that is typical of young adolescents. Several studies, particularly in the West and developed regions, indicate that marriage is protective [28-31], while others in many developing countries indicate that it is a significant source of abuse and stress, particularly for the women, leading to higher psychiatric morbidity, suicidal ideation and suicide [9,32-39]. Our study did not find marriage to be protective of suicide for women of all ages. In addition, we found non-fatal suicidal behavior rates to be higher in adolescents who were involved in unskilled labor compared to those who were students. Psychological, familial, social, and cultural factors risk factors that have been identified to influence adolescents to commit suicide include low socio-economic and educational status, family history of suicide, parental separation, divorce, or death, poor relationship with family and peers, social contagion, prevalence of psychiatric disorder, psychological stressors, sexual abuse, substance abuse, social deprivation, and availability of high lethality methods (e.g., guns) [27,40-42]. Another emerging issue is the media representation of suicidal behavior including the modern internet that have been found to have substantial influence on the vulnerable including adolescents in some Asian countries $[40,43]$.

Although not significant, the rates of fatal and non-fatal suicidal behavior were very high among agricultural workers and unskilled labor. Compared to skilled laborers students were, however, found to have lower rates of non-fatal suicidal behavior. This needs further attention and research since several studies have indicated that acute life stressors and poor educational status are significantly associated with higher rates of fatal and non-fatal suicidal behavior $[5,21,44,45]$. A variation was also seen geographically where fatal and non-fatal suicidal rates were found to be more in Sirajganj and Narsingdi districts of the country indicating the need to provide attention to particular socio-demographic or other factors in the regions.

Studies reporting methods of suicide indicate that availability of and accessibility to methods influence the choice of the method and act as factor in increasing overall fatal and non-fatal suicidal events [46]. As such, the common suicide methods not only vary by age and sex but also shift with the availability/restriction of new methods and technologies [47]. Globally, ingestion of pesticide, hanging and firearms are among the most common methods of suicide, whereas poisoning and hanging has been found to be most common in Asia $[2,5,7,47,48]$. Our study findings are consistent with those in Asia and reports hanging followed by poisoning to be the most common methods for suicide. For non-fatal suicidal behavior, the most common method was poisoning followed by hanging. Rural economy in Bangladesh is predominantly agricultural where availability of pesticides is rampant and unregulated. Several studies have indicated that, despite some challenges, means restriction through legal regulations, safe storage, awareness could be an important strategy for controlling fatal and non-fatal suicidal behavior at the population level $[43,46,49,50]$. For example, restrictions on the import and sales of pesticides in Sri Lanka resulted in reductions in suicide in both men and women of all ages with 19,769 fewer suicides occurring in 1996-2005 compared to 1986-1995 [51]. In addition to this research on suicide prevention also indicates the need for national strategy and community based approaches focusing on targeted groups $[52,53]$. The application of findings of prevention studies from other countries should be interpreted with caution given the unique sociocultural context of Bangladesh, and implementation research on suicide prevention in the country is required.

\section{Limitations and Challenges}

The study was conducted in rural Bangladesh and might not be generalizable for the country as risk factors might be very different in urban Bangladesh. Our study findings are generalizable to rural 
Bangladesh and similar settings in other low and middle income countries The fatal and non-fatal suicide rates, especially for non-fatal suicidal behavior, may be underreported due to challenges in data collection, recall bias, stigmatization and for punitive reasons. Given that information obtained from the head of the households may be subject to recall and social desirability biases, and to avoid stigma and likely legal punishments, suicide (especially of children and adolescent females) may be underreported, leading to biased fatal and nonfatal suicide rates. Contemporaneous and real-time death review/social autopsy may provide less biased estimates. Although mental illness is widely accepted as a risk factor of fatal and non-fatal suicidal behavior, our study was not designed to elicit any information on mental illness.

\section{Conclusions}

This study has made an attempt to provide updated information on the burden of suicide and suicidal attempts from a population census that covered almost 1.2 million rural people in five districts of the country. We conclude that suicide is a serious public health problem in Bangladesh especially among the high-risk individuals such as adolescent women and married women; some of the factors associated with completed and attempted suicide are similar or vary compared to other Asian and LMIC countries. Considering the context, where resources and services for suicide identification, treatment and support are limited, there is a need to develop targeted national strategies and action plans, prevention programs and conduct further research to learn more about the constraints and reduce the rate of suicide and its associated risk factors. Strategies to uplift the status of women in rural Bangladesh, such as creating opportunities that would improve financial independence, programs to create awareness of mental health issues and access to mental health treatment among married adolescent women, are among a few potential paths to take to address the huge public health burden of suicide. Further research to adequately characterize the burden of suicide and the gender disparities in suicide and attempted suicide rates in the country are needed. Implementation research on community-based prevention strategies, such as counseling/support groups and mental health awareness, is also needed to support the development of a national strategy.

There is also an urgent need to establish reliable systems to continuously collect data on suicide in Bangladesh that will help interested agencies to measure the social and economic burden needed to drive the establishment and implementation of effective suicide prevention programs in the country.

Supplementary Materials: The following are available online at www.mdpi.com/1660-4601/14/9/1032/s1, Table S1: Unadjusted and adjusted analysis of suicide among participants aged 10-17 year olds by sociodemographic and geographical factors, rural Bangladesh. Table S2: Unadjusted and adjusted analysis of attempted suicide among participants aged 10-17 year olds by socio-demographic and geographical factors, rural Bangladesh.

Acknowledgments: We would like to thank Bloomberg Philanthropies for providing the funding to implement the Saving of Lives from Drowning (SoLiD) study. We would also like to thank our local partners, Center for Injury Prevention and Research, Bangladesh (CIPRB) and icddr,b for their invaluable expertise and support in helping us implement the project.

Author Contributions: Shumona Sharmin Salam and Olakunle Alonge conceived the paper, contributed to the data analysis, wrote the initial drafts of the manuscript, and reviewed the final draft for intellectual content. Shams El Arifeen, Dewan Md Emdadul Hoque, Irteja Islam, Shumona Sharmin Salam, and Kamran Ul Baset contributed to the data collection and reviewed the final draft of the manuscript for intellectual content. Shirin Wadhwaniya and Saidur Rahman Mashreky reviewed the final draft of the manuscript for intellectual content. All co-authors provided editing support in finalizing the manuscript.

Conflicts of Interest: The authors declare no conflict of interest. The funding sponsors had no role in the design of the study; in the collection, analyses, or interpretation of data; in the writing of the manuscript, and in the decision to publish the results. 


\section{Abbreviations}

The following abbreviations are used in this manuscript:

$\begin{array}{ll}\text { LMIC } & \text { Low and Middle Income Countries } \\ \text { HIC } & \text { High Income Countries } \\ \text { CIPRB } & \text { Center for Injury Prevention and Research, Bangladesh } \\ \text { icddr,b } & \text { International Centre for Diarrhoeal Disease Research, Bangladesh (icddr,b) } \\ \text { JHSPH } & \text { John's Hopkins School of Public Health } \\ \text { CI } & \text { Confidence Interval } \\ \text { IRR } & \text { Incidence Rate Ratio } \\ \text { PYO } & \text { Person Years Observed }\end{array}$

\section{References}

1. WHO Estimates for 2000-2012. Available online: http://www.who.int/healthinfo/global_burden_disease/ estimates/en/ (accessed on 12 July 2016).

2. WHO Preventing Suicide: A Global Imperative. Available online: http://www.who.int/mental_health/ suicide-prevention/world_report_2014/en/ (accessed on 12 July 2016).

3. WHO Disease and Injury Country Estimates. Available online: http://www.who.int/healthinfo/global_ burden_disease/estimates_country/en/ (accessed on 7 July 2016).

4. Värnik, P. Suicide in the World. Int. J. Environ. Res. Public Health 2012, 9, 760-771. [CrossRef] [PubMed]

5. Chen, Y.Y.; Wu, K.C.C.; Yousuf, S.; Yip, P.S.F. Suicide in Asia: Opportunities and Challenges. Epidemiol. Rev. 2012, 34, 129-144. [CrossRef] [PubMed]

6. The WHO World Mental Health Surveys-Cambridge University Press. Available online: http: / / www.cambridge. org / catalogue / catalogue.asp?isbn=9780521884198 (accessed on 7 July 2016).

7. Jordans, M.J.; Kaufman, A.; Brenman, N.F.; Adhikari, R.P.; Luitel, N.P.; Tol, W.A.; Komproe, I. Suicide in South Asia: A Scoping Review. BMC Psychiatry 2014, 14, 1-9. [CrossRef] [PubMed]

8. Wang, C.W.; Chan, C.L.W.; Yip, P.S.F. Suicide Rates in China from 2002 to 2011: An Update. Soc. Psychiatry Psychiatr. Epidemiol. 2014, 49, 929-941. [CrossRef] [PubMed]

9. Patel, V.; Ramasundarahettige, C.; Vijayakumar, L.; Thakur, J.; Gajalakshmi, V.; Gururaj, G.; Suraweera, W.; Jha, P. Suicide Mortality in India: A Nationally Representative Survey. Lancet 2012, 379, 2343-2351. [CrossRef]

10. Mashreky, S.R.; Rahman, F.; Rahman, A. Suicide Kills More Than 10,000 People Every Year in Bangladesh. Arch. Suicide Res. 2013, 17, 387-396. [CrossRef] [PubMed]

11. GHO by Category Suicide Rates-Data by Country. Available online: http://apps.who.int/gho/data/view. main.MHSUICIDEv (accessed on 7 July 2016).

12. Alam, N.; Chowdhury, H.R.; Das, S.C.; Ashraf, A.; Streatfield, P.K. Causes of Death in Two Rural Demographic Surveillance Sites in Bangladesh, 2004-2010: Automated Coding of Verbal Autopsies Using InterVA-4. Glob. Health Action 2014. [CrossRef] [PubMed]

13. Nahar, Q.; Arifeen, S.E.; Jamil, K.; Streatfield, P.K. Causes of Adult Female Deaths in Bangladesh: Findings from Two National Surveys. BMC Public Health 2015. [CrossRef] [PubMed]

14. Hyder, A.A.; Alonge, O.; He, S.; Wadhwaniya, S.; Rahman, F.; Rahman, A.; Arifeen, S.E. Saving of Children's Lives from Drowning Project in Bangladesh. Am. J. Prev. Med. 2014, 47, 842-845. [CrossRef] [PubMed]

15. Hyder, A.A.; Alonge, O.; He, S.; Wadhwaniya, S.; Rahman, F.; Rahman, A.; Arifeen, S.E. A Framework for Addressing Implementation Gap in Global Drowning Prevention Interventions: Experiences from Bangladesh. J. Health Popul. Nutr. 2014, 32, 564-576. [PubMed]

16. Alonge, O.; Agarwal, P.; Taleb, A.; Rahman, Q.; Rahman, A.F.; Arifeen, S.E.; Hyder, A. Fatal and Non-Fatal Injury Outcomes: Results from a Purposively Sampled Census of Seven Rural Sub-Districts in Bangladesh. Lancet Glob. Health 2017, 5, 818-827. [CrossRef]

17. Fontanella, C.A.; Hiance-Steelesmith, D.L.; Phillips, G.S.; Bridge, J.A.; Lester, N.; Sweeney, H.A.; Campo, J.V. Widening Rural-Urban Disparities in Youth Suicides, United States, 1996-2010. JAMA Pediatr. 2015, 169, 466-473. [CrossRef] [PubMed]

18. Hirsch, J.K. A Review of the Literature on Rural Suicide: Risk and Protective Factors, Incidence, and Prevention. Crisis 2006, 27, 189-199. [CrossRef] [PubMed] 
19. Judd, F.; Cooper, A.M.; Fraser, C.; Davis, J. Rural Suicide-People or Place Effects? Aust. N.Z. J. Psychiatry 2006, 40, 208-216. [PubMed]

20. Möller-Leimkühler, A.M. The Gender Gap in Suicide and Premature Death or: Why Are Men so Vulnerable? Eur. Arch. Psychiatry Clin. Neurosci. 2003, 253, 1-8. [CrossRef] [PubMed]

21. Chau, K.; Kabuth, B.; Chau, N. Gender and Family Disparities in Suicide Attempt and Role of Socioeconomic, School, and Health-Related Difficulties in Early Adolescence. BioMed Res. Int. 2014, 2014, 1-13. [CrossRef] [PubMed]

22. Aggarwal, S. Suicide in India. Br. Med. Bull. 2015, 114, 127-134. [CrossRef] [PubMed]

23. Parker, G.; Yap, H.L. Suicide in Singapore: A Changing Sex Ratio over the Last Decade. Singap. Med. J. 2001, 42, 11-14.

24. Hadi, A. Risk Factors of Violent Death in Rural Bangladesh, 1990-1999. Death Stud 2005, 29, 559-572. [CrossRef] [PubMed]

25. Yusuf, H.R.; Akhter, H.H.; Chowdhury, M.E.; Rochat, R.W. Causes of Death among Women Aged 10-50 Years in Bangladesh, 1996-1997. J. Health Popul. Nutr. 2007, 25, 302-311. [PubMed]

26. Gipson, J.D.; Hicks, A.L.; Gultiano, S.A. Gendered Differences in the Predictors of Sexual Initiation among Young Adults in Cebu, Philippines. J. Adolesc. Health Off. Publ. Soc. Adolesc. Med. 2014, 54, 599-605. [CrossRef] [PubMed]

27. Silenzio, V.M.B.; Pena, J.B.; Duberstein, P.R.; Cerel, J.; Knox, K.L. Sexual Orientation and Risk Factors for Suicidal Ideation and Suicide Attempts Among Adolescents and Young Adults. Am. J. Public Health 2007, 97, 2017-2019. [CrossRef] [PubMed]

28. Masocco, M.; Pompili, M.; Vichi, M.; Vanacore, N.; Lester, D.; Tatarelli, R. Suicide and Marital Status in Italy. Psychiatr. Q. 2008, 79, 275-285. [CrossRef] [PubMed]

29. Corcoran, P.; Nagar, A. Suicide and Marital Status in Northern Ireland. Soc. Psychiatry Psychiatr. Epidemiol. 2009, 45, 795-800. [CrossRef] [PubMed]

30. Cutright, P.; Stack, S.; Fernquist, R. Marital Status Integration, Suicide Disapproval, and Societal Integration as Explanations of Marital Status Differences in Female Age-Specific Suicide Rates. Suicide Life Threat. Behav. 2007, 37, 715-724. [CrossRef] [PubMed]

31. Lorant, V.; Kunst, A.E.; Huisman, M.; Bopp, M.; Mackenbach, J. A European Comparative Study of Marital Status and Socio-Economic Inequalities in Suicide. Soc. Sci. Med. 2005, 60, 2431-2441. [CrossRef] [PubMed]

32. Khan, M.M. Suicide on the Indian Subcontinent. Crisis 2002, 23, 104-107. [CrossRef] [PubMed]

33. Khan, M.M. Suicide Prevention and Developing Countries. J. R. Soc. Med. 2005, 98, 459-463. [CrossRef] [PubMed]

34. Naved, R.T.; Akhtar, N. Spousal Violence against Women and Suicidal Ideation in Bangladesh. Womens Health Issues 2008, 18, 442-452. [CrossRef] [PubMed]

35. Devries, K.; Watts, C.; Yoshihama, M.; Kiss, L.; Schraiber, L.B.; Deyessa, N.; Heise, L.; Durand, J.; Mbwambo, J.; Jansen, H.; et al. Violence against Women Is Strongly Associated with Suicide Attempts: Evidence from the WHO Multi-Country Study on Women's Health and Domestic Violence against Women. Soc. Sci. Med. 2011, 73, 79-86. [CrossRef] [PubMed]

36. Pico-Alfonso, M.A.; Garcia-Linares, M.I.; Celda-Navarro, N.; Blasco-Ros, C.; Echeburúa, E.; Martinez, M. The Impact of Physical, Psychological, and Sexual Intimate Male Partner Violence on Women's Mental Health: Depressive Symptoms, Posttraumatic Stress Disorder, State Anxiety, and Suicide. J. Womens Health 2006, 15, 599-611. [CrossRef] [PubMed]

37. Seedat, S.; Stein, M.B.; Forde, D.R. Association between Physical Partner Violence, Posttraumatic Stress, Childhood Trauma, and Suicide Attempts in a Community Sample of Women. Violence Vict. 2005, 20, 87-98. [CrossRef] [PubMed]

38. Sudhir Kumar, C.T.; Mohan, R.; Ranjith, G.; Chandrasekaran, R. Gender Differences in Medically Serious Suicide Attempts: A Study from South India. Psychiatry Res. 2006, 144, 79-86. [CrossRef] [PubMed]

39. Maselko, J.; Patel, V. Why Women Attempt Suicide: The Role of Mental Illness and Social Disadvantage in a Community Cohort Study in India. J. Epidemiol. Community Health 2008, 62, 817-822. [PubMed]

40. Hawton, K.; Saunders, K.E.; O'Connor, R.C. Self-Harm and Suicide in Adolescents. Lancet 2012, 379, 2373-2382. [CrossRef]

41. Pitman, A.; Krysinska, K.; Osborn, D.; King, M. Suicide in Young Men. Lancet 2012, 379, 2383-2392. [CrossRef] 
42. Séguin, M.; Renaud, J.; Lesage, A.; Robert, M.; Turecki, G. Youth and Young Adult Suicide: A Study of Life Trajectory. J. Psychiatr. Res. 2011, 45, 863-870. [CrossRef] [PubMed]

43. Yip, P.S.; Caine, E.; Yousuf, S.; Chang, S.S.; Wu, K.C.C.; Chen, Y.Y. Means Restriction for Suicide Prevention. Lancet 2012, 379, 2393-2399. [CrossRef]

44. Bálint, L.; Osváth, P.; Rihmer, Z.; Döme, P. Associations between Marital and Educational Status and Risk of Completed Suicide in Hungary. J. Affect. Disord. 2016, 190, 777-783. [CrossRef] [PubMed]

45. Poorolajal, J.; Rostami, M.; Mahjub, H.; Esmailnasab, N. Completed Suicide and Associated Risk Factors: A Six-Year Population Based Survey. Arch. Iran. Med. 2015, 18, 39-43. [PubMed]

46. Florentine, J.B.; Crane, C. Suicide Prevention by Limiting Access to Methods: A Review of Theory and Practice. Soc. Sci. Med. 2010, 70, 1626-1632. [CrossRef] [PubMed]

47. Wu, K.C.C.; Chen, Y.Y.; Yip, P.S.F. Suicide Methods in Asia: Implications in Suicide Prevention. Int. J. Environ. Res. Public Health 2012, 9, 1135-1158. [CrossRef] [PubMed]

48. Milner, A.; De Leo, D. Suicide Research and Prevention in Developing Countries in Asia and the Pacific. Bull. World Health Organ. 2010, 88, 795-796. [CrossRef] [PubMed]

49. WHO Reducing Access to Means of Suicide. Available online: http://www.who.int/mental_health/mhgap/ evidence/suicide/q7/en/ (accessed on 7 July 2016).

50. Nordentoft, M. Crucial Elements in Suicide Prevention Strategies. Prog. Neuropsychopharmacol. Biol. Psychiatry 2011, 35, 848-853. [CrossRef] [PubMed]

51. Gunnell, D.; Fernando, R.; Hewagama, M.; Priyangika, W.D.D.; Konradsen, F.; Eddleston, M. The Impact of Pesticide Regulations on Suicide in Sri Lanka. Int. J. Epidemiol. 2007, 36, 1235-1242. [CrossRef] [PubMed]

52. Matsubayashi, T.; Ueda, M. The Effect of National Suicide Prevention Programs on Suicide Rates in 21 OECD Nations. Soc. Sci. Med. 2011, 73, 1395-1400. [CrossRef] [PubMed]

53. Fountoulakis, K.N.; Gonda, X.; Rihmer, Z. Suicide Prevention Programs through Community Intervention. J. Affect. Disord. 2011, 130, 10-16. [CrossRef] [PubMed]

(C) 2017 by the authors. Licensee MDPI, Basel, Switzerland. This article is an open access article distributed under the terms and conditions of the Creative Commons Attribution (CC BY) license (http:/ / creativecommons.org/licenses/by/4.0/). 